\title{
Effect of the extract from leaves of Liquidambar formosana Hance on S180 cells
}

\author{
Y.T. Zhong, X.L. Wang, Q.J. Xie and Y.N. Zhang \\ Gannan Medical University, Ganzhou, Jiangxi Province, China \\ Corresponding author: YT. Zhong \\ E-mail: zyt6869@126.com
}

Genet. Mol. Res. 15 (3): gmr.15038795

Received May 16, 2016

Accepted June 3, 2016

Published July 25, 2016

DOI http://dx.doi.org/10.4238/gmr.15038795

Copyright (C) 2016 The Authors. This is an open-access article distributed under the terms of the Creative Commons Attribution ShareAlike (CC BY-SA) 4.0 License.

\begin{abstract}
We examined the effects of the extract from leaves of Liquidambar formosana Hance on S180 cells and screened for antitumor active sites in the plant. Solvent extraction was conducted to prepare extracts from the leaves of $L$. formosana Hance and conduct preliminary separation, an MTT assay to determine the effect of leaf extract on the proliferation of S180 cells, and inverted microscopy to observe the effect of chloroform extract on the morphology of S180 cells. Double-staining (Annexin V/propidium iodide) with flow cytometry was conducted to determine the effect of the chloroform extract on S180 cell apoptosis. At some concentrations, the different extracts from the leaves of $L$. formosana Hance dose-dependently inhibited the proliferation of S180 cells. Among all extracts, the chloroform extract showed the strongest inhibitory effect on S180 cell proliferation. The $\mathrm{IC}_{50}$ values for the chloroform extract, ethyl acetate extract, $n$-butanol extract, and water layer were $0.238,0.471,0.844$, and $0.411 \mathrm{mg} / \mathrm{mL}$, respectively. We observed cell shrinkage, volume reduction, and varying sizes by inverted microscopy. Additionally, with increasing drug concentration, the number of cells decreased and debris
\end{abstract}


increased. The cells showed typical apoptotic morphological changes. The chloroform extract induced the apoptosis of S180 cells in a dosedependent manner. Different extracts from the leaves of L. formosana Hance inhibited the proliferation of S180 cells, and the chloroform extract was the main antitumor component. This extract from the leaves of $L$. formosana Hance inhibited the proliferation of S180 cells in part by inducing apoptosis.

Key words: S180 cells; Leaves of Liquidambar formosana Hance; Active sites; Apoptosis

\section{INTRODUCTION}

Liquidambar formosana Hance is also known as maple, nienie, and acer buergerianum, among other names. It belongs to the Hamamelidaceae liquidambar deciduous trees and is common in warm, humid climates. This species is mainly distributed in the Qinling Mountains, Huaihe, south of the Pearl River Basin. All parts of liquidambar can be used as medicinal materials. The leaves are fragile, have a clear aroma when rubbed, and have weinxin and a slightly bitter flavor (Chen, 2000; Huang et al., 2000).

The leaves of $L$. formosana Hance are used as heat antidotes, to arrest diarrhea, and to treat dysentery, treat pain, heatstroke, postpartum wind, and pediatric tetanus neonatorium (Jiangshu New Medical College, 1977). The leaves of L. formosana Hance are a very safe and natural source of melanin, which is not only a pigment, but also important in medicine and health care. The leaves of $L$. formosana Hance are often used as a food coloring pigment material. Additionally, L. formosana Hance leaves can be boiled and mashed for use in the soaking of glutinous rice, which is black and shiny with good taste (Chen, 1998). Previous studies showed that the leaves of L. formosana Hance have many pharmacological activities; they can be used to treat diabetes and blood pressure and contain essential trace elements for humans (Xie et al., 2000; Huang and Song, 2001; Zhong et al., 2010). They also have antibacterial effects (Zheng et al., 2005; Zhong et al., 2007) and can inhibit the proliferation of human leukemia K562 cells (Xie et al., 2015) as well as improve non-specific immune function and cellular immune function in normal mice (Zhong et al., 2012). In this study, we evaluated the effects of different solvent extracts prepared from the leaves of L. formosana Hance on S180 cells to identify its anti-tumor active sites.

\section{MATERIAL AND METHODS}

\section{Instruments}

Continuous spectrum multi-function enzyme (Varioskan Flash, Thermo Fisher, Waltham, MA, USA), inverted phase contrast microscope (CKX 41, Olympus, Tokyo, Japan), flash extractor (HBE-50S, EYELA), rotary evaporator (N-1000v-W, Büchi, Flawil, Switzerland), atmospheric microwave/extraction reactor (WF-4000C, EYELA), flow cytometry (FACS Calibur, BD Biosciences, Franklin Lakes, NJ, USA), clean bench (SW-CJ2FD, Boxun, Shanghai, China), and $\mathrm{CO}_{2}$ incubator (Forma 311, Thermo Fisher) were used in this study.

Genetics and Molecular Research 15 (3): gmr.15038795 


\section{Materials}

The murine sarcoma cell line S180 was obtained from the Institute of Medicine, Chinese Academy of Medical Sciences. Leaves of L. formosana Hance were collected at the Huangjin campus of Gannan Medical University of Jiangxi province in the month of June. Professor Li Jialin, from the Department of Pharmacy at Gannan Medical University, identified all leaves of L. formosana Hance. Ethanol (70\%), aether petrolei, chloroform, acetic ether, and watersaturated butanol were purchased from Guoyao Corporation (Nanjing, China). Other materials included dimethyl sulfoxide (Sigma, St. Louis, MO, USA), DMEM (Gibco, Grand Island, NY, USA), fetal calf serum (Si Jiqing Co., Hangzhou, China), Annexin V/propidium iodide (PI, BD Biosciences), and 3-(4,5-dimethylthigal-2-yl)-2,5-(diphenyltetragalium) bromide (MTT, Sigma) were used.

\section{Methods}

\section{Preparation of experimental drugs}

First, $200 \mathrm{~g}$ of washed and dried leaves of L. formosana Hance was mashed and mixed with $70 \%$ ethanol twice for extraction. Each extraction was performed for $1 \mathrm{~h}$. The filtrate together and decompre and recover alcohol extract, extract was mixed by water and extracted with petroleum ether, chloroform, ether acetate, and normal butanol in the same volume in turn. After evaporating the extraction and water layers, we obtained petroleum ether extract, chloroform extract, ethyl acetate extract, $n$-butyl alcohol extract, and water layer. This dry paste was dissolved in dimethyl sulfoxide and its volume fraction was less than $0.1 \%$. Phosphate-buffered saline volumes were very low and could not be formulated as $100 \mathrm{mg} / \mathrm{mL}$ storage solution (solubility of the extract of petroleum ether extract is very low and cannot be formulated as the concentration required); therefore, it was not used in the experiment, and the samples were stored at $4^{\circ} \mathrm{C}$. Samples were prepared to the required concentration when used.

\section{Cell culture}

S180 cells were inoculated into the abdominal cavity of Kunming mice; after seeing obvious ascites, we injected the cells into other mice. After stably passaging the cells for 3-4 generations, we obtained the ascites, centrifuged the washed S180 cells, and stained the cells with trypan blue. If more than $95 \%$ cells were S180 cells, we conducted the MTT experiments.

\section{Effects of different extracts from leaves of L. formosana Hance on the proliferation of S180 cells by MTT}

Cells in the logarithmic growth phase were adjusted to $1 \times 10^{5} / \mathrm{mL}$ in DMEM culture medium containing $10 \%$ fetal bovine serum and incubated in 96 -well plates $(180 \mu \mathrm{L}$ per well). The samples were then placed at $37^{\circ} \mathrm{C}, 5 \% \mathrm{CO}_{2}$, and saturated humidity and incubated for $18 \mathrm{~h}$. We divided the cells into a blank control group and drug experimental group, placing enough cell groups in 6 wells. We added $20 \mu \mathrm{L}$ PBS to the blank control group and $20 \mu \mathrm{L}$ 
different concentrations $(0.25,0.5,1,2,4,8$, and $16 \mathrm{mg} / \mathrm{mL}$, all concentrations were based on preliminary test results) extract from leaves of $L$. formosana Hance in the drug experimental group, and then continued to cultivate the cells for $24 \mathrm{~h}$. Inverted microscopy was used to record morphology; $20 \mu \mathrm{L}$ MTT $(5 \mathrm{mg} / \mathrm{mL})$ was added to each well and the plate was incubated for $4 \mathrm{~h}$ and centrifuged at $1500 \mathrm{rpm} / \mathrm{min}$ for $10 \mathrm{~min}$. The supernatant was removed and $150 \mu \mathrm{L}$ dimethyl sulfoxide was added to each well, concussed, and mix evenly for $10 \mathrm{~min}$, after which the absorbance of A (optical density, OD) was measured using a microplate spectrophotometer at $570 \mathrm{~nm}$. The inhibitory rate and the median inhibition concentration of the drug to the tumor cells was calculated, and we evaluated the preliminary efficacy of the drug using the following formula: tumor cell inhibition rate $(\%)=(1$ - experimental group OD)/blank control group OD $\times 100 \%$. $\log C$ - inhibitory rate regression analysis was used to calculate the median inhibition concentration $\left(\mathrm{IC}_{50}\right)$.

\section{Morphological observation}

The chloroform extract of the leaves of $L$. formosana Hance was added to S180 cells. The processes of dividing the cells into groups, concentrations, and times were the same as in the MTT experiment. The growth and changes in cell morphology of S180 cells in the experimental and control groups were observed by inverted microscopy and photos were acquired.

\section{Detection of $\mathbf{S 1 8 0}$ cell apoptosis induced by chloroform extract with Annexin V-fluorescein isothiocyanate/PI double-staining method}

Cells in the logarithmic growth phase were adjusted to $2 \times 10^{5} / \mathrm{mL}$ and incubated in 6-well plates $\left(1.8 \mathrm{~mL}\right.$ per well), then placed in a $37^{\circ} \mathrm{C}, 5 \% \mathrm{CO}_{2}$, and saturated humidity for $18 \mathrm{~h}$. We divided the cells into a solvent control group and drug experimental group, added $200 \mu \mathrm{L}$ experimental drug (chloroform extract was 0.25 and $0.5 \mathrm{mg} / \mathrm{mL}$ ) into the experimental group, added the same volume of PBS into the control group, incubated the cells for $24 \mathrm{~h}$, and then harvested the cells. We blew cells into a single cell suspension. According to the kit instructions, using V-fluorescein isothiocyanate/PI double-staining and flow cytometry to detect cell apoptosis and calculated the percentage of total cells and apoptotic cells based on Annexin V-positive staining (AV+/PI-).

\section{RESULTS}

\section{Effect of different extracts from liquidambar leaves on S180 cell proliferation}

The results are shown Table 1; these cells were treated with the extracts for $24 \mathrm{~h}$ at different concentrations. Different extracts from the leaves of $L$. formosana Hance inhibited the proliferation of S180 cells in a dose-dependent manner. The inhibition rate of S180 cells and $\mathrm{IC}_{50}$ were chloroform extract $>$ water layer $>$ ethyl acetate extract $>$ N-butanol extract, with chloroform extract showing the strongest inhibitory effect on S180 cells proliferation. Thus, the chloroform extract from the leaves of L. formosana Hance contained the main active components with antitumor activity, and therefore we selected chloroform extract for subsequent analysis.

Genetics and Molecular Research 15 (3): gmr.15038795 
Table 1. Inhibition rate of the proliferation of $\mathrm{S} 180$ cells and $\mathrm{IC}_{50}$ after treatment with different extracts from the leaves of Liquidambar formosana Hance after $24 \mathrm{~h}$.

\begin{tabular}{l|c|c|c|c}
\hline Group & Chloroform extract & Ethyl acetate extract & N-butanol extract & Water layer \\
\hline Control & 0 & 0 & 0 & 0 \\
\hline $0.025 \mathrm{mg} / \mathrm{mL}$ & $4.15 \pm 2.53$ & $-34.39 \pm 8.74$ & $4.96 \pm 4.18$ & $2.65 \pm 4.06$ \\
\hline $0.05 \mathrm{mg} / \mathrm{mL}$ & $15.34 \pm 2.97$ & $3.94 \pm 4.75$ & $8.41 \pm 5.11$ & $6.70 \pm 3.13$ \\
\hline $0.1 \mathrm{mg} / \mathrm{mL}$ & $29.38 \pm 1.06$ & $18.84 \pm 3.14$ & $13.66 \pm 13.80$ & $26.44 \pm 9.69$ \\
\hline $0.2 \mathrm{mg} / \mathrm{mL}$ & $51.07 \pm 0.90$ & $31.51 \pm 2.32$ & $25.19 \pm 3.49$ & $36.17 \pm 0.07$ \\
\hline $0.4 \mathrm{mg} / \mathrm{mL}$ & $78.08 \pm 1.12$ & $53.90 \pm 1.35$ & $37.40 \pm 2.12$ & $55.50 \pm 0.19$ \\
\hline $0.8 \mathrm{mg} / \mathrm{mL}$ & $81.50 \pm 0.82$ & $64.14 \pm 2.34$ & $49.32 \pm 1.24$ & $68.09 \pm 0.31$ \\
\hline $1.6 \mathrm{mg} / \mathrm{mL}$ & $82.18 \pm 0.96$ & $74.89 \pm 1.58$ & $60.37 \pm 0.81$ & $75.03 \pm 0.59$ \\
\hline $\mathrm{IC} 50 \mathrm{mg} / \mathrm{mL}$ & 0.238 & 0.471 & 0.844 & 0.411 \\
\hline
\end{tabular}

Effect of chloroform extract from leaves of $L$. formosana Hance on morphology of S180 cells

As shown in Figure 1, S180 cells in the control group grew rapidly as clusters; the cells were large, round, and bright, and a large number of cells were actively dividing. The growth of S180 cells was inhibited by treatment with chloroform extract for $24 \mathrm{~h}$. Cells were in the growth state of dispersion and shrinkage, and some cells shrunk and were different sizes. With increasing drug concentration, the number of cells decreased and cell debris increased. The cells showed typical apoptotic morphological changes (Figure 1).
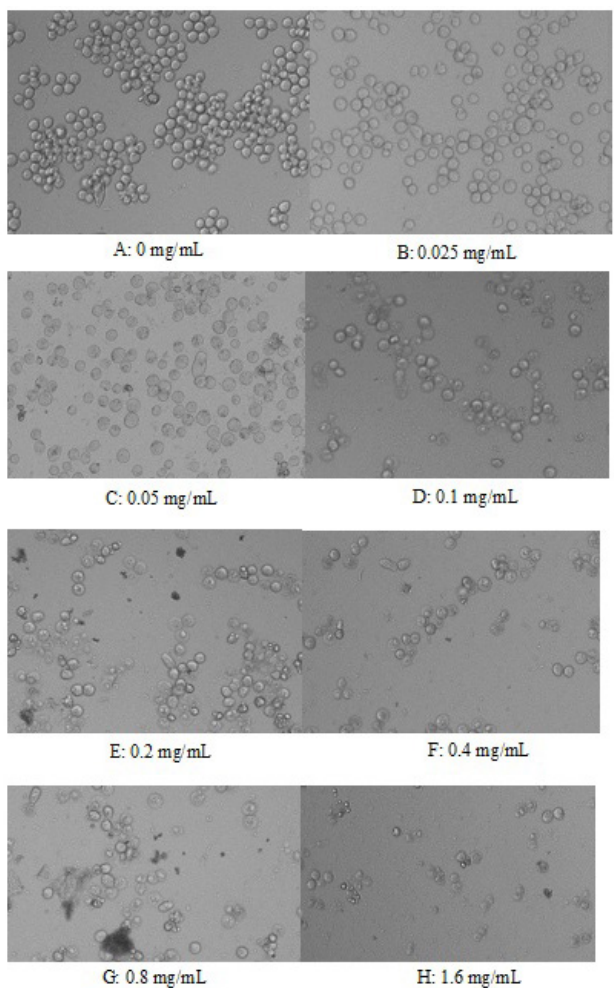

Figure 1. Effect of chloroform extract from leaves of Liquidambar formosana Hance on the morphology of S180 cells after $24 \mathrm{~h}(200 \mathrm{X})$.

Genetics and Molecular Research 15 (3): gmr.15038795 


\section{Effect of chloroform extract from leaves of $L$. formosana Hance on S180 cell apoptosis}

As shown in Figure 2, we determined the effect of different concentrations of chloroform extract on S180 cells after $24 \mathrm{~h}$. We detected the apoptosis rate by Annexin V/PI doublestaining. The results showed that the apoptosis rate was significantly increased with increasing drug concentration. When the drug concentration was $0.025 \mathrm{mg} / \mathrm{mL}$, the early apoptosis rate was $3.88 \%$, while the late apoptosis rate was $3.86 \%$. When the drug concentration was 0.05 $\mathrm{mg} / \mathrm{mL}$, the early apoptosis rate and late apoptosis rate were 4.05 and $4.21 \%$, respectively. Compared with the control group, these differences were significant $(\mathrm{P}<0.05)$.

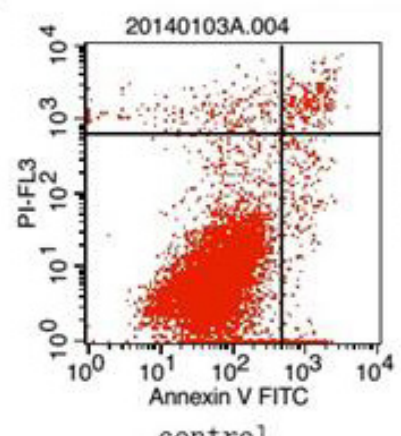

control

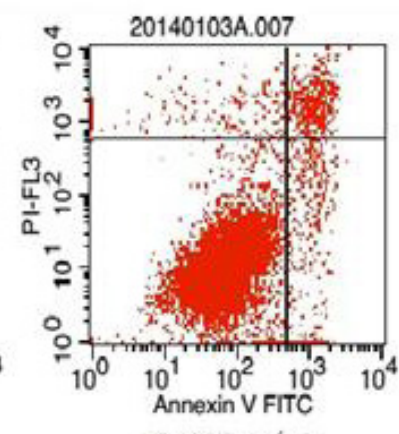

$0.025 \mathrm{mg} / \mathrm{ml}$

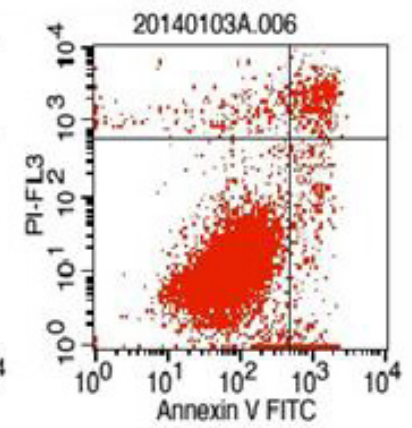

$0.05 \mathrm{mg} / \mathrm{ml}$

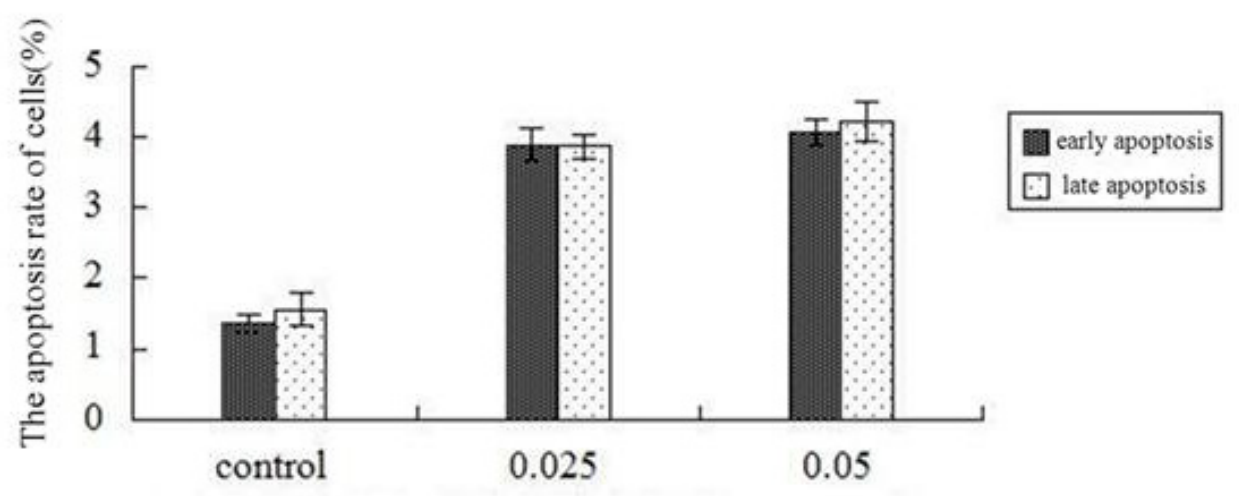

Figure 2. Effect of chloroform extract from leaves of Liquidambar formosana Hance on S180 cell apoptosis.

\section{DISCUSSION}

In recent years, Chinese herbal medicine and natural plants have played important roles in tumor therapy. Herbal medicine and natural plant medicine have relatively minor toxic side effects, unique antitumor effects, and good curative effects. They have received increased attention and have been examined for their ability to prevent and cure tumors and for the development of antitumor drugs (Wang et al., 2010).

Genetics and Molecular Research 15 (3): gmr.15038795 
The leaves of $L$. formosana Hance contain terpenoids, flavonoids, tannins, and volatile oil (Rong, 2003). Previous studies reported that terpenoids, flavonoids, and tannins have anti-tumor effects (Xu et al., 2008). The antitumor mechanism of terpenoids occurs mainly through the inhibition of tumor cell proliferation and the induction of tumor cell apoptosis, anti-angiogenesis, and anti-invasion (Lee et al., 2011). Flavonoids resist tumors by inhibiting tumor cell proliferation, inducing apoptosis, regulating the cell cycle, interfering with cell signal transduction, and inhibiting angiogenesis (Cao et al., 2004).

According to the principle of similarity law, chloroform can be used to extract terpenoids, while the ethyl acetate layer can extract alkaloids, flavonoids, and tannins. These substances inhibit the proliferation of tumor cells. However, the content and activity of the extract affect the antitumor effects of the extract. We found that at certain concentrations, the extract of chloroform, ethyl acetate, $n$-butanol, and water from the leaves of $L$. formosana Hance could inhibit the proliferation of S180 cells in a dose-dependent manner. The inhibition rate increased with increasing drug concentration, and the chloroform extract showed the strongest inhibitory effect on S180 cell proliferation, which was the principal antitumor active fraction. The chloroform extract from the leaves of $L$. formosana Hance was used to treat S180 cells for $24 \mathrm{~h}$, and cell proliferation was shown to be inhibited. Cells were in the growth state of dispersion and shrinkage, and the cells shrunk and were different sizes. With increasing drug concentration, the number of cells decreased and cell debris increased. The cells showed typical apoptotic morphological changes. The apoptosis rate was detected by Annexin V/PI double-staining. The results showed that the apoptosis rate was significantly increased with increasing drug concentration. When the drug concentration was $0.025 \mathrm{mg} / \mathrm{mL}$, the early apoptosis rate was $3.88 \%$ and the late apoptosis rate was $3.86 \%$. When the drug concentration was $0.05 \mathrm{mg} / \mathrm{mL}$, the early apoptosis rate and the late apoptosis rate were 4.05 and $4.21 \%$, respectively. Compared to the control group, these differences were statistically significant ( $\mathrm{P}$ $<0.05$ ). These results demonstrate that the chloroform extract from the leaves of $L$. formosana Hance induced S180 cell apoptosis. One of the mechanisms of inhibition of the leaf extract of L. formosana Hance on S180 cell proliferation is to induce cell apoptosis.

The chemical constituents and antitumor mechanism of Chinese herbal medicine are very complex. Single components and mechanisms can play a role, but a variety of components and mechanisms are also involved. The effect of crude extracts from the leaves of L. formosana Hance on S180 cells was explored. The exact chemical composition and antitumor mechanism require further analysis.

\section{REFERENCES}

Cao LQ, Wang XL and Liu DY (2004). Advances of study on apoptosis of tumor cells induced by flavonoids. Zhong Yao Cai 27: 785-788.

Chen DC (2000). Xian Dai Shi Yong Ben Cao. 2nd Vol. People's Medical Publishing, Beijing.

Chen ZH (1998). Study on extraction of melanin from the leaves of Liquidambar formosana Hance. Lin Chan Hua Gong Tong Xun 1: 20-21.

Huang L and Song XP (2001). Determination and analysis of inorganic elements essential to human in Hainan Liquidambar formosana. Stud. Trace Elements Health 18: 45-46.

Huang TK, Ding ZZ and Zhao SX (2000). Xian Dai Ben Cao Gang Mu.1st volume. China Press of Traditional Chinese Medicine, Beijing.

Jiangshu New Medical College (1977). Zhong Yao Da Ci Dian. 1st Vol. Shanghai People's Publishing House, Shanghai.

Lee TK, Castilho A, Cheung VC, Tang KH, et al. (2011). Lupeol targets liver tumor-initiating cells through phosphatase and tensin homolog modulation. Hepatology 53: 160-170. http://dx.doi.org/10.1002/hep.24000

Genetics and Molecular Research 15 (3): gmr.15038795 
Rong R (2003). Advances of study on chemical constituents and pharmacological activities of plants of Liquidambar formosana Hance. China News Trad. Chin. Med. 1929: 4-9.

Wang LM, Jiang QL, Bi SY, Shi WG, et al. (2010). Study on the inhibitory effect of hyperoside on the proliferation of tumor cells in vitro. Heilongjiang Med. Pharm. 33: 73-74.

Xie QJ, Xu XY, Zheng JR and Zhong YT (2015). Study on effect of extract from the leaves of Liquidambar formosana Hance on K562 cells. Chin. Med. Mat. 7: 1493-1495.

Xie YS, Zheng XJ and Zheng LR (2000). Analysis of some water-soluble trace elements in the leaves of Liquidambar formosana Hance. J. Guangxi Teachers Coll. 17: 5-7.

Xu XD, Hu XR and Yang JS (2008). Study on the effective components of antitumor medicinal plants. J. Chin. Mat. Med. 33: 2073-208.

Zheng Y, Liu NF, Xiao WH, Zhong Q, et al. (2005). Study on preventing effect of extract from the leaves of Liquidambar formosana Hance on pepper black spot. J. Jiangxi Agric. Univ. 27: 96-98.

Zhong YT, Wang L, Wang XL and Sun XT (2010). Determination of trace elements in the leaves of Liquidambar formosana Hance by flame atomic absorption spectrometry. LiShiZhen Med. Mat. Med. Res. 21: 1457-1458.

Zhong YT, Wang XL and Ma LL (2007). Study on antibacterial activity of the leaves of Liquidambar formosana Hance. LiShiZhen Med. Mat. Med. Res. 18: 1693-1694.

Zhong YT, Wang XL, Sun XT, Xu J, et al. (2012). Study on the effect of extract from the leaves of Liquidambar formosana Hance on mouse immune function. Pharmacol. Clin. Chin. Mat. Med. 28: 124-126.

Genetics and Molecular Research 15 (3): gmr.15038795 